\title{
Intratumoral concentration of estrogens and clinicopathological changes in ductal carcinoma in situ following aromatase inhibitor letrozole treatment
}

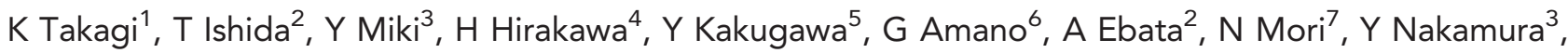 \\ M Watanabe ${ }^{8}, \mathrm{M} \mathrm{Amari}^{2}$, N Ohuchi ${ }^{2}, \mathrm{H}$ Sasano ${ }^{3,8}$ and T Suzuki ${ }^{*}, 1$
}

${ }^{1}$ Department of Pathology and Histotechnology, Tohoku University Graduate School of Medicine, 2-1 Seiryo-machi, Aoba-ku, 980-8575 Sendai, Japan; ${ }^{2}$ Department of Surgical Oncology, Tohoku University Graduate School of Medicine, 2-1 Seiryo-machi, Aoba-ku, 980-8575 Sendai, Japan; ${ }^{3}$ Department of Anatomic Pathology, Tohoku University Graduate School of Medicine, 2-1 Seiryo-machi, Aoba-ku, 980-8575 Sendai, Japan; ${ }^{4}$ Department of Surgery, Tohoku Kosai Hospital, 2-3-11 Kokubun-cho, Aoba-ku, 980-0803 Sendai, Japan; ${ }^{5}$ Department of Breast Oncology, Miyagi Cancer Center Hospital, Medeshimashiote, 47-1 Nodayama, 981-1239 Natori, Japan; ${ }^{6}$ Department of Surgery, Nihonkai General Hospital, 30 Akiho, 998-8501 Sakata, Japan; ${ }^{7}$ Department of Diagnostic Radiology, Tohoku University Graduate School of Medicine, 2-1 Seiryo-machi, Aoba-ku, 980-8575 Sendai, Japan and ${ }^{8}$ Department of Pathology, Tohoku University Hospital, Aoba-ku, 1-1 Seiryo-machi, 980-9574 Sendai, Japan

Background: Estrogens have important roles in ductal carcinoma in situ (DCIS) of the breast. However, the significance of presurgical aromatase inhibitor treatment remains unclear. Therefore, we examined intratumoral concentration of estrogens and changes of clinicopathological factors in DCIS after letrozole treatment.

Methods: Ten cases of postmenopausal oestrogen receptor (ER)-positive DCIS were examined. They received oral letrozole before the surgery, and the tumour size was evaluated by ultrasonography. Surgical specimens and corresponding biopsy samples were used for immunohistochemistry. Snap-frozen specimens were also available in a subset of cases, and used for hormone assays and microarray analysis.

Results: Intratumoral oestrogen levels were significantly lower in DCIS treated with letrozole compared with that in those without the therapy. A great majority of oestrogen-induced genes showed low expression levels in DCIS treated with letrozole by microarray analysis. Moreover, letrozole treatment reduced the greatest dimension of DCIS, and significantly decreased Ki-67 and progesterone receptor immunoreactivity in DCIS tissues.

Conclusion: These results suggest that estrogens are mainly produced by aromatase in DCIS tissues, and aromatase inhibitors potently inhibit oestrogen actions in postmenopausal ER-positive DCIS through rapid deprivation of intratumoral estrogens.

The incidence of ductal carcinoma in situ (DCIS) has been markedly increasing during the past two decades, and now represents $25-30 \%$ of all mammographically detected breast cancers (Chen et al, 2009). Ductal carcinoma in situ has a $14-53 \%$ risk of invasive carcinoma over a period of $\geqslant 10$ years after biopsy (Erbas et al, 2006), and it is currently treated with simple mastectomy or breast-conserving surgery, with or without radiation therapy, to prevent local recurrence (Burstein et al, 2004; Dixon et al, 2007). On the other hand, survival rate of DCIS is $\sim 98-99 \%$ (Burstein et al, 2004), and it is also important to 
consider minimising of aggressive treatments in DCIS patients (Chen et al, 2009).

Estrogens have an important role in the development and progression of invasive breast carcinoma. Estrogens are locally synthesised in the invasive breast carcinoma tissues from circulating inactive steroids by oestrogen-producing enzymes, such as aromatase (conversion from circulating androstenedione to estrone or testosterone to estradiol), steroid sulphatase (hydrolysis of circulating estrone sulphate to estrone) and $17 \beta$-hydroxysteroid dehydrogenase type 1 (17 $\beta$ HSD1; conversion of estrone to estradiol) (Suzuki et al, 2005). Oestrogen actions are mediated through oestrogen receptor (ER), and endocrine therapies, such as aromatase inhibitors (i.e., letrozole, anastrozole and exemestane) and tamoxifen, are used in patients with ER-positive invasive carcinoma to block the oestrogen actions. Results of recent clinical trials demonstrated that presurgical aromatase inhibitor treatment for postmenopausal women with ER-positive invasive breast cancer reduced the tumour volume, and the conversion rate from mastectomy to breast-conserving surgery was $48-88 \%$ and was superior to tamoxifen (Takei et al, 2011).

Intratumoral production of estrogens has also been demonstrated in DCIS tissues (Shibuya et al, 2008). Also, a great majority of DCIS expressed ER (Wiechmann and Kuerer, 2008). These findings indicate the importance of oestrogen actions in DCIS, and aromatase inhibitors might be effective for postmenopausal women with ER-positive DCIS as well as invasive carcinoma. Neoadjuvant aromatase inhibitor therapy in DCIS patients has been reported only by two groups (Chen et al, 2009; Bundred et al, 2010), to the best of our knowledge. These were immunohistochemical studies, and they showed significant decrease in Ki-67 and progesterone receptor (PR) immunoreactivity in ER-positive DCIS tissues after the treatment. On the other hand, aromatase expression in DCIS has been reported to be significantly lower than in invasive carcinoma (Shibuya et al, 2008), and importance of aromatase has not yet been fully clarified in DCIS. No information is available regarding effects of aromatase inhibitor on intratumoral oestrogen levels, molecular oestrogen actions or tumour size in DCIS, and biological and/or clinical significance of aromatase inhibitor thus remains largely unclear in DCIS. Therefore, in this study, we first examined intratumoral concentration of estrogens in DCIS with letrozole treatment. Moreover, we examined expression profiles of oestrogen-induced genes and several clinicopathological factors in DCIS tissues after letrozole treatment, and compared these findings with those at baseline.

\section{MATERIALS AND METHODS}

Patients and tissues. This study was a non-randomized uncontrolled trial, and registered at University hospital Medical Information Network Clinical Trial Registry (UMIN-CTR) (http://www.umin.ac.jp/ctr/index/htm) (ID: UMIN000003101). The main inclusion criteria was the diagnosis of ER-positive DCIS in postmenopausal patients by biopsy, and letrozole treatment was performed before the surgery in these patients.

Ten postmenopausal women with ER-positive DCIS were enrolled in this study; they underwent breast-conserving surgery from 2010 to 2011 at Tohoku University Hospital (Sendai, Japan), Tohoku Kosai Hospital (Sendai, Japan), Miyagi Cancer Center Hospital, (Natori, Japan), and Nihonkai General Hospital (Sakata, Japan) (range of age; 52-74). All patients gave written informed consent. The patients' characteristics are summarised in Table 1. They received oral letrozole (Femara; Novartis Pharma (Tokyo, Japan)), $2.5 \mathrm{mg}$ daily for 3-27 weeks before breastconserving surgery. Information about the tumour size evaluated by ultrasonography (US) was available in nine cases both before

\begin{tabular}{|c|c|c|c|c|c|}
\hline Case & Age & $\begin{array}{c}\text { Menopausal } \\
\text { status }\end{array}$ & $\begin{array}{c}\text { Diagnosis } \\
\text { at } \\
\text { baseline }\end{array}$ & $\begin{array}{l}\text { Diagnosis } \\
\text { after the } \\
\text { letrozole } \\
\text { treatment }\end{array}$ & $\begin{array}{c}\text { Period } \\
\text { of the } \\
\text { letrozole } \\
\text { treatment } \\
\text { (weeks) }\end{array}$ \\
\hline 1 & 69 & Post & $\mathrm{DClS}(\mathrm{ER}+)$ & DCIS & 15 \\
\hline 2 & 57 & Post & $\mathrm{DClS}(\mathrm{ER}+)$ & $\mathrm{DClS}$ & 14 \\
\hline 3 & 74 & Post & DCIS (ER+) & $\begin{array}{c}\text { IDC } \\
(\mathrm{pT} 1 \mathrm{mic} \\
(<1 \mathrm{~mm}))\end{array}$ & 14 \\
\hline 4 & 52 & Post & $\mathrm{DClS}(\mathrm{ER}+)$ & $\mathrm{DClS}$ & 4 \\
\hline 5 & 57 & Post & $\mathrm{DClS}(\mathrm{ER}+)$ & $\mathrm{DClS}$ & 3 \\
\hline 6 & 62 & Post & $\mathrm{DClS}(\mathrm{ER}+)$ & $\mathrm{DClS}$ & 7 \\
\hline 7 & 68 & Post & $\mathrm{DClS}(\mathrm{ER}+)$ & DCIS & 10 \\
\hline 8 & 58 & Post & $\mathrm{DCIS}(\mathrm{ER}+)$ & $\begin{array}{c}\text { IDC } \\
(\mathrm{pT1a}(2 \mathrm{~mm}))\end{array}$ & 27 \\
\hline 9 & 67 & Post & $\mathrm{DClS}(\mathrm{ER}+)$ & DCIS & 14 \\
\hline 10 & 72 & Post & $\mathrm{DCIS}(\mathrm{ER}+)$ & $\begin{array}{c}\text { IDC } \\
(\text { pT1mic } \\
(<1 \mathrm{~mm}))\end{array}$ & 14 \\
\hline
\end{tabular}

and after letrozole treatment in their charts. The entire resected surgical specimen was sectioned into slices with 3-5 mm thickness, and all the slices were histologically evaluated. At post-treatment diagnosis, seven cases were diagnosed as pure DCIS, whereas small invasive foci were detected in three cases. In all the cases, both biopsy at baseline and surgical specimens were available in the formalin-fixed and paraffin-embedded tissues. Snap-frozen specimens were also available in a subset of cases, and these specimens were used for hormone assays (baseline samples: $n=2$, post-treatment samples: $n=8$ ) and/or microarrary analysis (baseline samples: $n=2$, post-treatment samples: $n=5$ ).

As untreated controls for hormone assay, six snap-frozen specimens of ER-positive DCIS were also used in this study. These were obtained from postmenopausal women who underwent surgical treatment from 2001 to 2004 at Tohoku University Hospital and Tohoku Kosai Hospital, Sendai, Japan. These patients did not receive any neoadjuvant therapy.

The research protocol was approved by the Ethics Committee at Tohoku University School of Medicine and other institutional review boards.

Liquid chromatography/electrospray tandem mass spectrometry (LC-MS/MS). Concentrations of estrone, estradiol and androstenedione were measured by LC-MS/MS analysis in ASKA Pharma Medical Co., Ltd (Kawasaki, Japan), as described previously (Takagi et al, 2013a). Briefly, tissue specimens were homogenised in $1 \mathrm{ml}$ of distilled water, and steroid fraction was extracted with diethyl ether. In this study, we used an LC (Agilent 1100, Agilent Technologies, Waldbronn, Germany) coupled with an API 4000 triple-stage quadrupole mass spectrometer (Applied Biosystems, Ontario, Canada) operated with electron spray ionisation in the positive-ion mode, and the chromatographic separation was performed on Cadenza CD-C18 column $(3 \times 150 \mathrm{~mm}, 3.5 \mathrm{~mm}$, Imtakt, Kyoto, Japan). In our present study, the lower limits of quantification (LLOQ) of estrone, estradiol and androstenedione were $0.05,0.03$ and $2.0 \mathrm{pg}$ per assay, respectively. 
LASER capture microdissection (LCM)/microarray analysis. Gene expression profiles of breast carcinoma cells were examined using microarray analysis. LCM was conducted using the MMI Cellcut (Molecular Machines and Industries, Glattbrugg, Switzerland). Briefly, breast carcinomas were embedded in Tissue-Tek optimal cutting temperature compound (Sakura Finetechnical Co., Tokyo, Japan) and sectioned at a thickness of $10 \mu \mathrm{m}$. Breast carcinoma cells were dissected under the light microscopy and laser transferred from these frozen sections. The total RNA ( $\sim 200 \mathrm{ng}$ ) was subsequently extracted from these cell fractions isolated by LCM using the RNeasy Micro Kit (QIAGEN, Mannheim, Germany). SurePrint G3 Hmn GE $8 \times 60$ K Microarray Kit (G4851A, ID 028004; Agilent Technologies) was used in this study, and sample preparation and processing were performed according to the manufacturer's protocol.

In this study, we focused on the expression profiles of two gene lists that were previously reported as oestrogen-induced genes in invasive breast carcinoma cell line MCF-7 (i.e., Frasor's (Frasor et al, 2003) and Creighton's (Creighton et al, 2006) lists that consisted of 50 and 63 genes, respectively). Among these, two genes in Frasor's list and three genes in Creighton's list were not available in our present study. If a gene was represented multiple times on the platform, the probe with strongest positive correlation with ESR1 (ER) was selected (Takagi et al, 2013a).

Immunohistochemistry. Monoclonal antibodies for ER (6F11), progesterone receptor (PR; 1A6), and Ki-67 (MIB-1) were purchased from NovoCastra (Newcastle upon Tyne, UK), Chemicon (Temecula, CA, USA), and DAKO (Carpinteria, CA, USA) respectively. Monoclonal antibody for aromatase (clone \#677/H7) was kindly provided by Dr Evans DB, Novartis, Basel, Switzerland. Rabbit polyclonal antibodies for HER2 (A0485) were purchased from DAKO, C-MYB (EPR718(2)) and RBAP46 (EPR5082) from Epitomics (Burlingame, CA, USA), and survivin (NB500-201) from Novus Biologicals (Littleton, CO, USA).

A Histofine Kit (Nichirei Biosciences, Tokyo, Japan), which employs the streptavidin-biotin amplification method, was used in this study. Antigen retrieval was performed by heating the slides in an autoclave at $120^{\circ} \mathrm{C}$ for $5 \mathrm{~min}$ in antigen retrieval solution ( $\mathrm{pH}$ 9.0) (Nichirei Biosciences) for C-MYB immunostaining or citric acid buffer $(2 \mathrm{~mm}$ citric acid and $9 \mathrm{~mm}$ trisodium citrate dehydrate ( $\mathrm{pH}$ 6.0)) for immunostaining of ER, PR, HER2, Ki-67, RBAP46 and survivin. Dilutions of primary antibodies used in this study were as follows: ER, 1/50; PR, 1/50; HER2; 1/100; Ki-67, 1/100; aromatase, 1/3000; C-MYB, 1/50; RBAP46, 1/1000 and survivin, $1 / 1000$. The antigen-antibody complex was subsequently visualised with $3,3^{\prime}$-diaminobenzidine (DAB) solution (1 mM DAB, $50 \mathrm{~mm}$ Tris- $\mathrm{HCl}$ buffer $\left(\mathrm{pH}\right.$ 7.6), and $0.006 \% \mathrm{H}_{2} \mathrm{O}_{2}$ ) and counterstained with hematoxylin.

Immunoreactivity of ER, PR Ki-67, C-MYB and RBAP46 was detected in the nucleus, and their immunoreactivity was evaluated in counting $>1000$ carcinoma cells for each case. The percentage of immunoreactivity, that is, labelling index (LI), was subsequently determined. Cases with ER LI of $>10 \%$ were considered ERpositive breast carcinoma in this study. Aromatase immunoreactivity was detected in the cytoplasm, and the cases that had $>10 \%$ of positive carcinoma cells were considered positive (Takagi et al, 2010). HER2 immunoreactivity was evaluated according to the grading system proposed in HercepTest (DAKO), and strongly circumscribed membrane-immunoreactivity of HER2 present in $>$ $30 \%$ carcinoma cells were considered positive (Wolff et al, 2007). Survivin immunoreactivity was detected in the cytoplasm of carcinoma cells, and was semi-quantitatively evaluated by modified LI system (Mehta et al, 2011), in which the percentage of cytoplasmic immunoreactivity was scored as 0 (no expression), 10 (up to 10\%), 20 (10-20\%) until 100 (90-100\%).

\section{RESULTS}

Intratumoral concentration of estrogens in DCIS tissues treated with letrozole. We first examined tissue concentration of sex steroids in DCIS treated with letrozole by LC-MS/MS. The median with min-max value of intratumoral estrone concentration was $0.23(0-1.1) \mathrm{pgg}^{-1}$ in DCIS tissues treated with letrozole, which was significantly lower $(0.0003$-fold and $P=0.0008)$ than that without the therapy (76.5 (11.9-159.3) $\mathrm{pgg}^{-1}$ ) (Figure 1A).

A

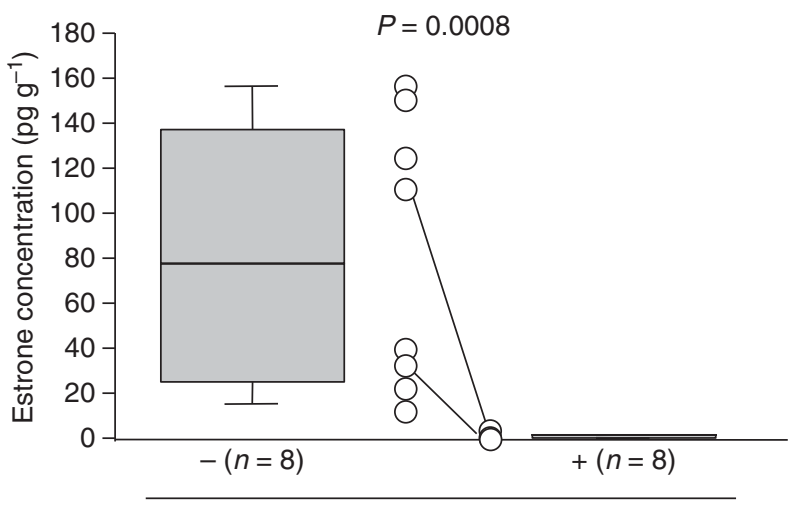

Letrozole treatment

B
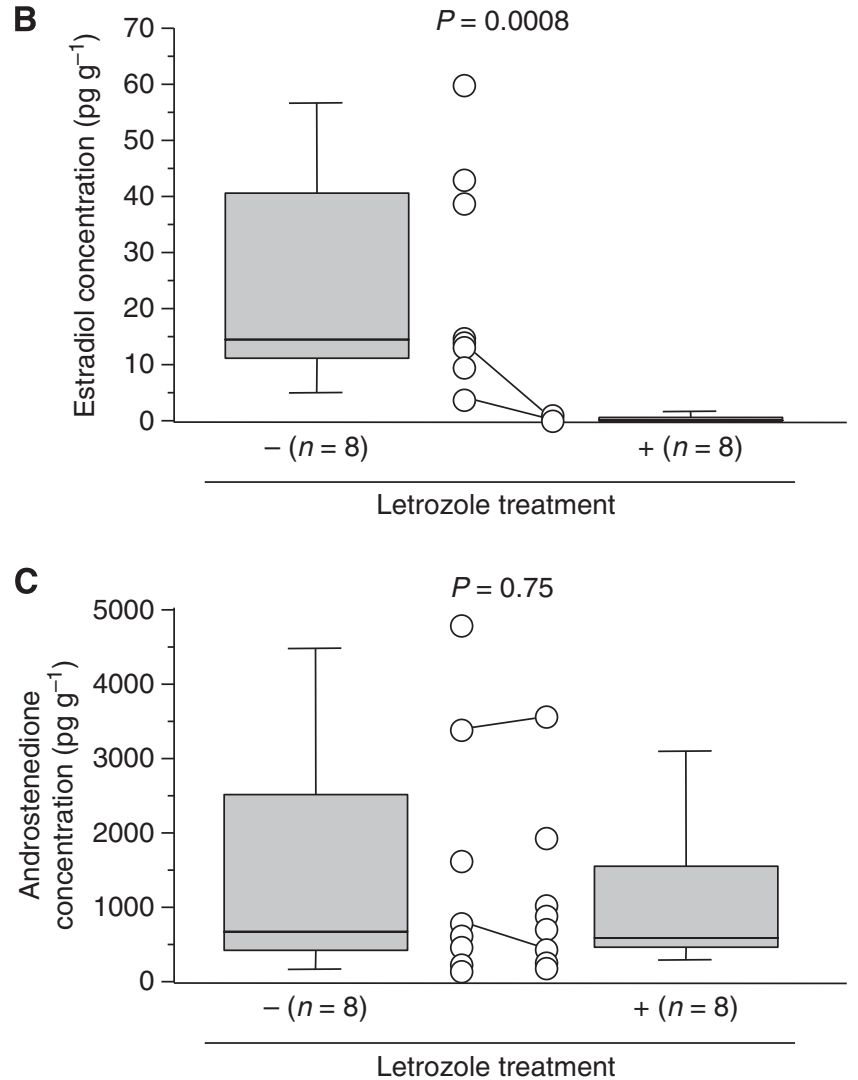

Figure 1. Tissue concentration of estrone (A), estradiol (B) and androstenedione (C) in DCIS with or without letrozole treatment using LC-MS/MS analysis. A horizontal line in the box pot illustrated the median value, and grey box denotes the 75th (upper margin) and 25th percentiles of the values (lower margin), respectively. The upper and lower bars indicate the 90th and 10th percentiles, respectively. Each value was shown in an open circle, and the paired values from the same patient were connected in a line $(n=2)$. The statistical analyses were performed using a Mann-Whitney U-test. P-values $<0.05$ were considered significant, and indicated in bold. 
Tissue concentration of estradiol was also significantly lower (0.015-fold and $P=0.0008)$ in DCIS treated with letrozole $(0.23$ $\left.(0-2.0) \mathrm{pg} \mathrm{g}^{-1}\right)$ than the untreated group (14.3 (3.1-62.7) $\left.\mathrm{pg} \mathrm{g}^{-1}\right)$ (Figure 1B). On the other hand, androstenedione level of DCIS was not significantly different $(P=0.75)$ between letrozole-treated (586 (228-3563) $\mathrm{pgg}^{-1}$ ) and untreated (687 (128-4864) $\mathrm{pg} \mathrm{g}^{-1}$ ) groups in this study (Figure 1C).

Expression profiles of oestrogen-induced genes in DCIS treated with letrozole. In order to examine the effect of letrozole treatment on molecular functions of estrogens in DCIS, we then performed microarray analysis in DCIS tissues, and compared the expression profiles of oestrogen-induced gene between baseline $(n=2)$ and post-treated $(n=5)$ DCIS tissues by scatter plot analysis. In this analysis, when the expression ratio of a gene in the letrozole-treated samples when compared with that in the baseline samples was $>2.0$ or $<0.5$, we determined higher or lower expression in the letrozole treatment, respectively. In the Frasor's list, 44 out of 48 (92\%) genes examined showed lower expression in the letrozole treatment group, and no gene showed higher expression compared with the baseline (Figure 2A). Paired samples at baseline and post-treatment were available in two cases (Case 4 and Case 8), and similar tendency was detected in each case (Figure 2B and C). When we used Creighton's list to confirm these findings, 58 out of 60 (97\%) genes showed lower expression in the post-treatment group (Figure 2D). The microarray data of Figure $2 \mathrm{~A}$ and D are provided in Supplementary Table S1.

Change of tumour size in DCIS patients after letrozole treatment. We next evaluated an association of various clinicopathological parameters in DCIS treated with letrozole, and compared these findings to their baseline. The greatest dimension of DCIS evaluated by US was decreased by $18 \%$ after the treatment (baseline: $11(6-22) \mathrm{mm}$ and post-treatment: 9 (3-28) $\mathrm{mm}$ ), although $P$-value did not reach a significant level $(P=0.17)$
(Figure 3A). When we classified the patients into two groups according the median value of the treatment duration (94 days), the tumour size was significantly (0.81-fold and $P=0.043$ ) decreased after treatment $(9(3-16) \mathrm{mm})$ compared with baseline (11 $(6-22) \mathrm{mm}$ ) in the longer treatment group (Figure $3 \mathrm{~B}$ ), whereas these were similar $(0.95$-fold and $P=0.85)$ in the shorter duration group. Relative tumour size after letrozole treatment compared with baseline was $27 \%$ smaller in the longer treatment group (73 (50-90) \%) when compared with the shorter group (100 $(50-255) \%)$, although it was not significant $(P=0.18)$ (Figure $3 C$ ).

DCIS lesions were also evaluated by magnetic resonance (MR) imaging after letrozole treatment in the seven patients. Among these, three cases showed higher signal intensity within the lesions compared with surrounding normal breast tissue on fat-suppressed T2-weighted MR imaging (Figure 3D).

Morphological and immunohistochemical features of DCIS after letrozole treatment. Morphologically, DCIS tissues after letrozole treatment frequently showed increased periductal fibrosis or infiltration of foam cells compared with their baseline, as report by Chen et al (2009) (Figure 4). Necrosis was not prevalent in DCIS tissues following letrozole treatment.

Associations of various pathological parameters in 10 paired DCIS tissues before and after letrozole treatment are summarised in Table 2. PR LI (Figure 5B) and Ki-67 LI (Figure 5C) were significantly decreased after letrozole treatment compared with the baseline $(P=0.018$ and $P=0.0050$, respectively). On the other hand, nuclear grade, comedo necrosis, ER LI (Figure 5A), HER2 and aromatase immunoreactivity (Figure 5D) were not changed after the letrozole treatment in this study.

Results of our microarray analysis demonstrated that a great majority of oestrogen-induced genes showed lower expression level in DCIS tissues after letrozole treatment. Among these genes examined, RBAP46, C-MYB and survivin were recently reported to be highly expressed in DCIS (Ebata et al, 2012). Therefore, we next
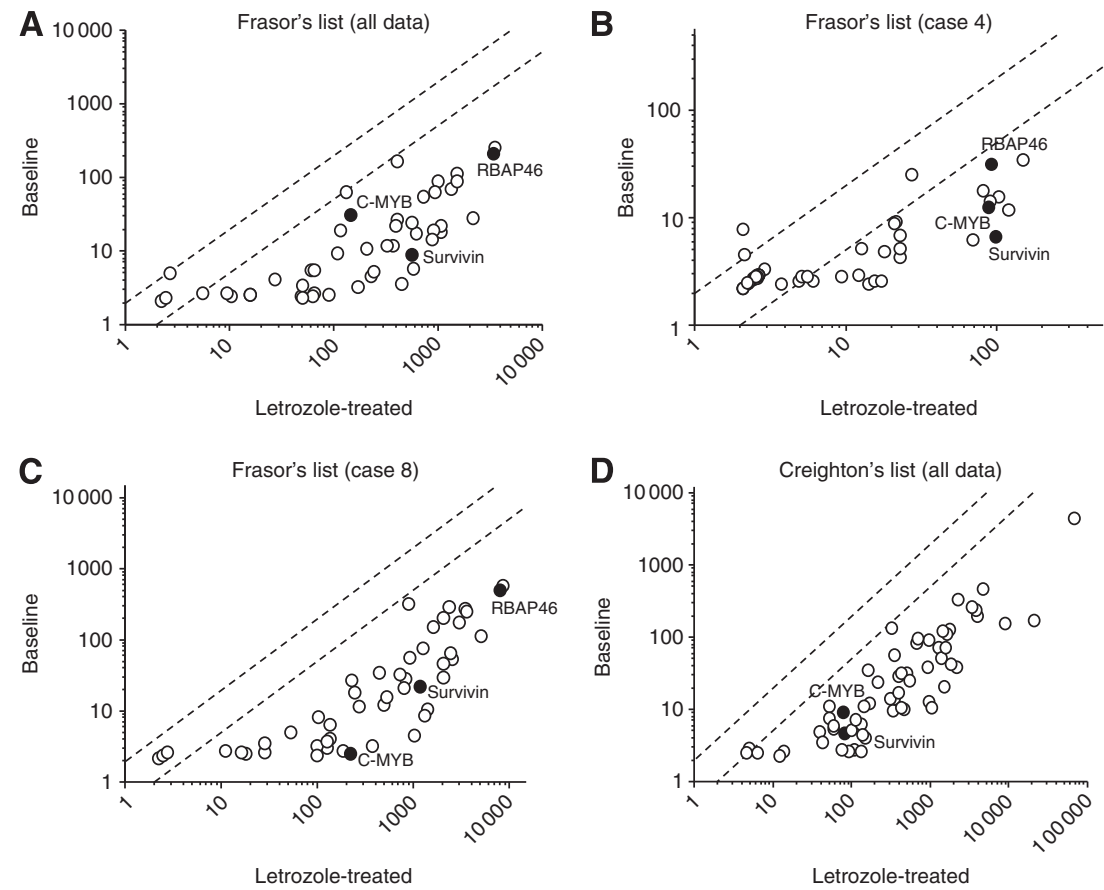

Figure 2. Scatter plot analysis of microarray data of estrogen-induced genes listed by Frasor et al (2003) (A-C) and Creighton et al (2006) (D). Genes with an expression ratio, post-treatment to baseline, of $>2.0$ or $<0.5$ are located outside the diagonal dotted line. A and $\mathbf{D}$ summarise all the values (baseline group; $n=2$, and post-treatment group; $n=5$ ), while $(\mathbf{B})$ and $(\mathbf{C})$ showed the paired data obtained from Case 4 and Case 8, respectively. Locations of estrogen-induced genes, for which immunohistochemistry was performed (i.e., RBAP46, C-MYB and survivin), were noted as closed circles. 

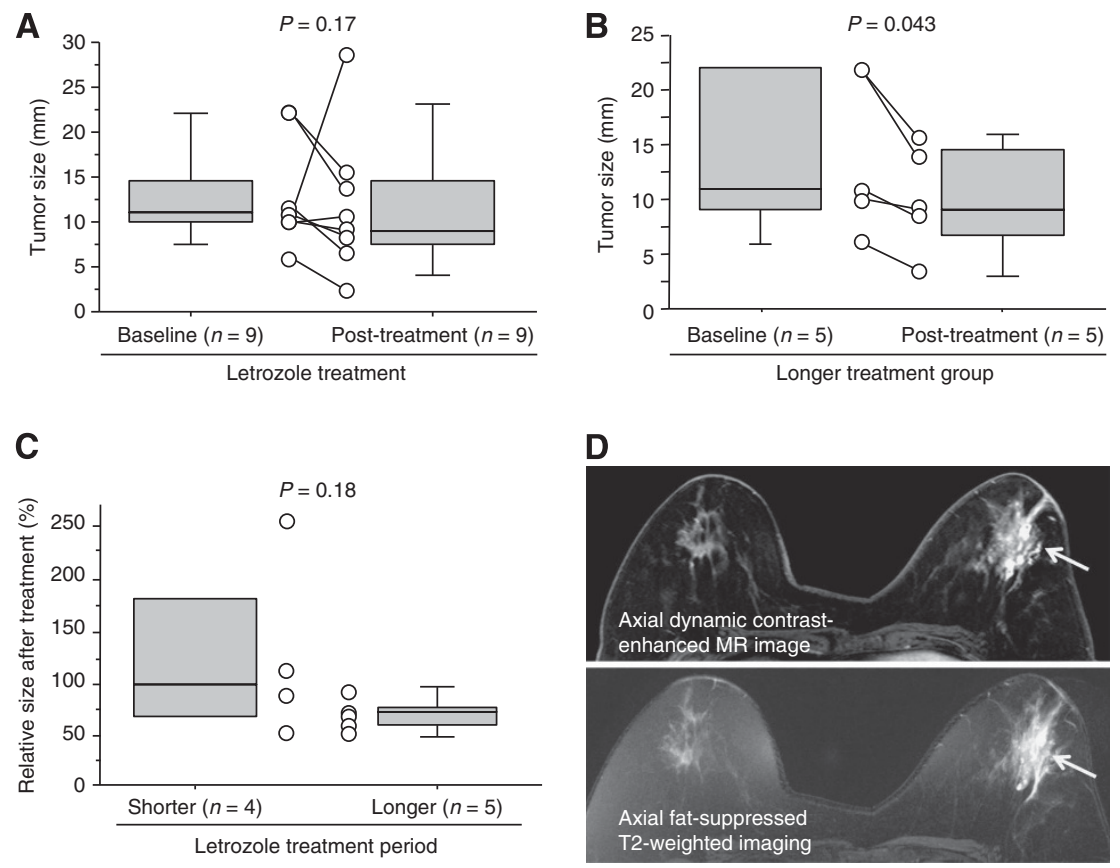

Figure 3. Clinical response of DCIS after letrozole treatment. (A-C) Tumor size of DCIS patients treated with letrozole evaluated by ultrasonography (US). The median value of the greatest dimension was illustrated by a horizontal line in the box pot, and grey box denotes the 75th (upper margin) and 25th percentiles of the values (lower margin), respectively. The upper and lower bars indicate the 90th and 10th percentiles, respectively. Each value was shown in an open circle, and the paired values from the same patient were connected in a line. The statistical analyses were performed using a Wilcoxon signed rank test (A, B), and a Mann-Whitney U-test (C). P-value $<0.05$ was considered significant, and indicated in bold. (D) Representative MR images after letrozole treatment (Case 5). Axial dynamic contrast-enhanced MR images obtained in early phase represented a segmental non-mass-like lesion in the left breast (arrow) (upper panel), and corresponding axial fat-suppressed T2-weighted imaging showed strong high signal intensity within the lesion (arrow), compared with that in the contralateral right breast (lower panel).

performed immunohistochemistry for these proteins to confirm the results of microarray analyses. As shown in Table 2, RBAP46 (Figure 5E), C-MYB (Figure 5F) and survivin (Figure 5G) LIs were significantly lower in DCIS tissues after the letrozole treatment ( $P=0.017, P=0.013$ and $P=0.017$, respectively).

Changes of PR and Ki-67 LIs between baseline and posttreatment samples were further examined according to several clinicopathological factors. As shown in Table 3, Ki-67 LI in the post-treatment group was significantly lower in a group positive for aromatase immunoreactivity at baseline $(P=0.035)$, but not in the aromatase-negative group $(P=0.46)$. Post-treatment diagnosis and period of letrozole treatment did not significantly change the PR or Ki-67 LI both at baseline and post-treatment in this study.

\section{DISCUSSION}

This is the first report to evaluate intratumoral oestrogen concentrations in DCIS tissues treated with aromatase inhibitor. In this study, median values of estrone and estradiol in DCIS treated with letrozole were 0.6 and $1.5 \%$ of those not treated with letrozole $(P=0.0008)$, respectively. Aromatase immunoreactivity in DCIS tissue was not changed after letrozole treatment in this study. Previous studies demonstrated that intratumoral oestrogen concentrations were markedly suppressed in invasive breast carcinoma treated with aromatase inhibitors. For instance, treatment with anastrozole for 15 weeks suppressed tissue estrone and estradiol levels by 83 and $89 \%$, respectively, compared with baseline levels (Geisler et al, 2001), and letrozole treatment for 16 weeks suppressed pretreatment tumour levels of estrone and estradiol by 91 and 98\%, respectively (Geisler et al, 2008). The results of our present study were in good agreement with these previous results, and suggest that intratumoral oestrogen concentrations of DCIS were remarkably deprived by aromatase inhibitor to a similar extent as in invasive carcinoma, nevertheless aromatase protein remained in the tissues.

Duration of letrozole treatment was variable from 3-27 weeks in our present study, but both estrone and estradiol concentrations in DCIS tissues were almost negligible regardless of the treatment period. Recently, we reported that invasive breast carcinoma tissues of patients who received exemestane treatment for 2 weeks maintained relatively high concentration of estradiol (35\% of that without this mode of therapy, and $P=0.56$ between these two groups), and speculated that other oestrogen-producing enzymes, such as steroid sulphatase and $17 \beta \mathrm{HSD} 1$, may interrupt the rapid decrease in the intratumoral estradiol level (Takagi et al, 2010). Although no data are currently available on the influence of shortterm letrozole therapy on estradiol concentration in the invasive carcinoma patients to the best of our knowledge, our present results in DCIS seem superior to those in the invasive carcinoma, and these may suggest that estrogens are mainly synthesised by aromatase in DCIS rather than other enzymes.

The results of our microarray analysis demonstrated that a great majority of oestrogen-induced genes (92\% in Frasor's list and 97\% in Creighton's list) showed $<50 \%$ expression levels in DCIS treated with letrozole compared with that in a group without this mode of therapy. We confirmed these results by employing immunohistochemistry for three representative genes (i.e., RBAP46, C-MYB and survivin). On the other hand, letrozole treatment did not significantly change ER LI in our study. The lists of oestrogen-induced genes used in this study were identified in 

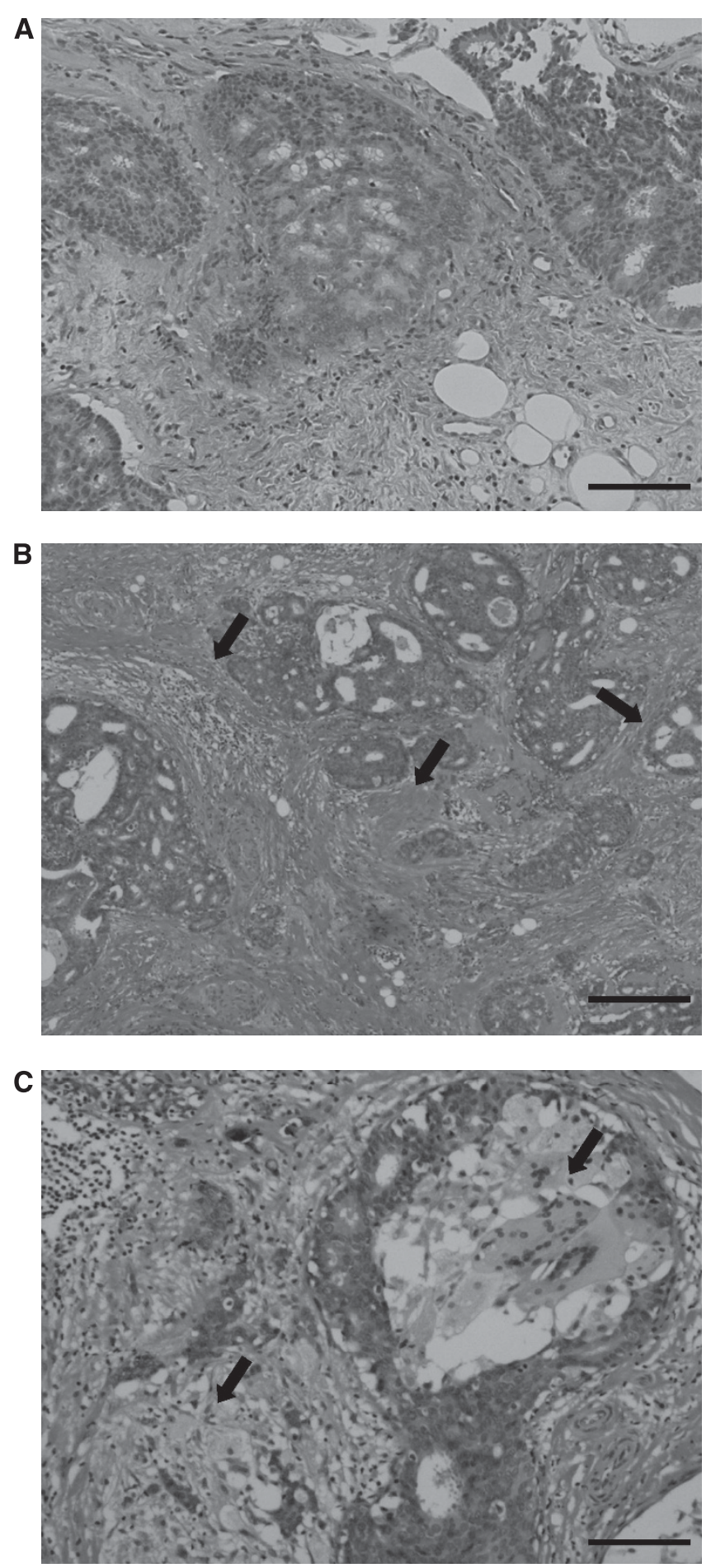

Figure 4. Morphological features of DCIS at baseline (A) and corresponding post-treatment (B, C) (Case 1). Increased periductal fibrosis (B) and infiltration of foam cells (C) were observed after the letrozole treatment as shown by arrows. Hematoxylin and eosin (HE) staining. Bar $=100 \mu \mathrm{m}$, respectively. A full colour version of this figure is available at the British Journal of Cancer journal online.

MCF-7 invasive breast carcinoma cells (Frasor et al, 2003; Creighton et al, 2006), as no information is available on oestrogen-induced genes in DCIS cells. The results of our study suggest that a great majority of oestrogen-induced genes identified in the invasive carcinoma cells are also regulated by estrogens in DCIS, and aromatase inhibitor broadly suppresses these expressions consistent with deprivation of intratumoral estrogens even if
Table 2. Change of pathological parameters in 10 paired DCIS tissues obtained before and after the letrozole treatment

\begin{tabular}{|l|c|c|c|}
\hline Parameter & Baseline & Post- treatment & P-value \\
\hline Nuclear grade & a \\
\hline Grades 1+2 & 8 & 8 & - \\
Grade 3 & 2 & 2 & $>0.99$ \\
\hline
\end{tabular}

\begin{tabular}{l|c|c|c|}
\hline \multicolumn{4}{|l|}{ Comedo necrosis $^{\mathbf{a}}$} \\
\hline Absent & 5 & 5 & - \\
Present & 5 & 5 & $>0.99$ \\
ER LI (\%) & $97(15-100)$ & $95(30-100)$ & $>0.99$ \\
PR LI (\%) & $93(0-100)$ & $18(0-83)$ & 0.018 \\
\hline
\end{tabular}

\section{HER2 status ${ }^{a}$}

\begin{tabular}{|l|c|c|c|}
\hline Negative & 9 & 9 & - \\
Positive & 1 & 1 & $>0.99$ \\
Ki-67 LI (\%) & $10(4-24)$ & $3(0-7)$ & 0.005 \\
\hline
\end{tabular}

\section{Aromatase immunoreactivity ${ }^{a}$}

\begin{tabular}{|l|c|c|c|}
\hline Negative & 5 & 5 & - \\
Positive & 5 & 5 & $>0.99$ \\
RBAP46 LI (\%) & $58(33-98)$ & $38(15-62)$ & 0.017 \\
C-MYB LI (\%) & $35(7-82)$ & $10(0-25)$ & 0.013 \\
Survivin LI (\%) & $30(0-80)$ & $20(0-40)$ & 0.017 \\
\hline
\end{tabular}

The statistical analyses were performed using a Wilcoxon signed rank test, and $P$-values $<0.05$ were considered significant, and given in bold.

${ }^{a}$ Data are presented as the number of cases, and all other values represent the median (min-max)

ER expression remains. Among the genes markedly suppressed by letrozole in this study, some were reported to be closely associated with cell proliferation (CCND1 (Steeg and Zhou, 1998) and BUB1 (Takagi et al, 2013b)) and invasion (EGR3 (Suzuki et al, 2007)) of the breast carcinoma, DCIS recurrence (survivin (Barnes et al, 2006)), and transition from DCIS to invasive carcinoma (MYC (Robanus-Maandag et al, 2003)), although biological functions remains largely unclear in a majority of oestrogeninduced genes. Various oestrogen functions, including cell proliferation, invasion and metastasis, are mediated by networks and pathways of the oestrogen-regulated genes (Frasor et al, 2003, Suzuki et al, 2012), and therefore, aromatase inhibitor may inhibit the growth and progression to invasive carcinoma in DCIS.

In our immunohistochemical study, letrozole treatment significantly decreased Ki-67 LI $(0.3$-fold and $P=0.0050)$ and PR LI (0.19-fold and $P=0.018$ ). Ki-67 LI is well known to reflect the proliferative activity of breast carcinoma (van Diest et al, 2004). On the other hand, PR positivity is considered to represent an intact oestrogen-signaling pathway (Dowsett et al, 2005) and often used as an indicator of a response to endocrine therapy in the invasive breast carcinoma (Bardou et al, 2003), although PR gene was not included in Frasor's or Creighton's lists. Previously, Chen et al (2009) reported that Ki-67 LI and PR score were significantly lower in DCIS tissues treated with letrozole for 3 months $(n=9)$ compared with that in the control group $(P=0.013$ and $P=0.001$, respectively), and Bundred et al (2010) also showed that exemestane treatment for 2 weeks significantly reduced Ki-67 and PR compared with placebo group in 22 DCIS patients $(P<0.001$ and $P=0.011$, respectively). Our present results are in good agreement with these previous studies.

Moreover, our results did demonstrate that Ki-67 LI after letrozole treatment was significantly lower $(0.25$-fold and $P=0.035)$ in aromatase-positive cases at baseline than in the negative group. Previous studies reported an association between 
A

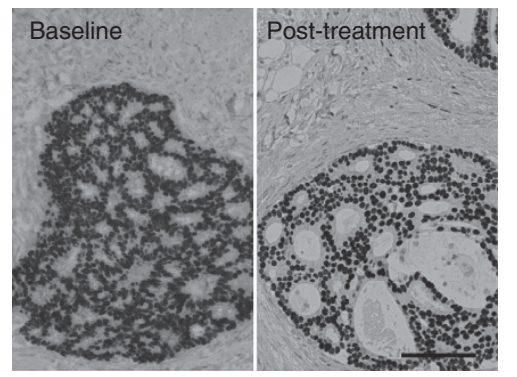

C

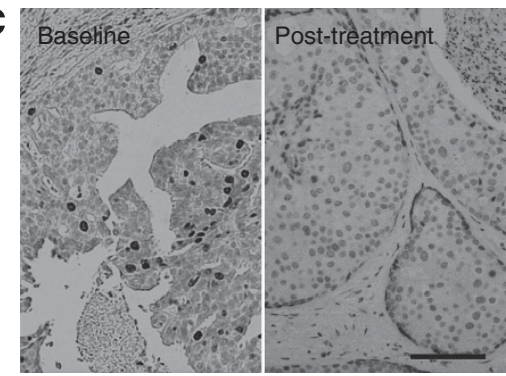

$\mathbf{E}$

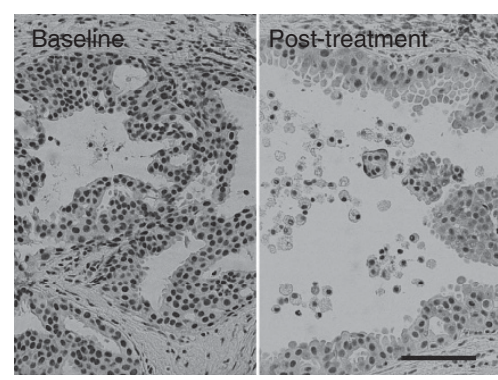

B
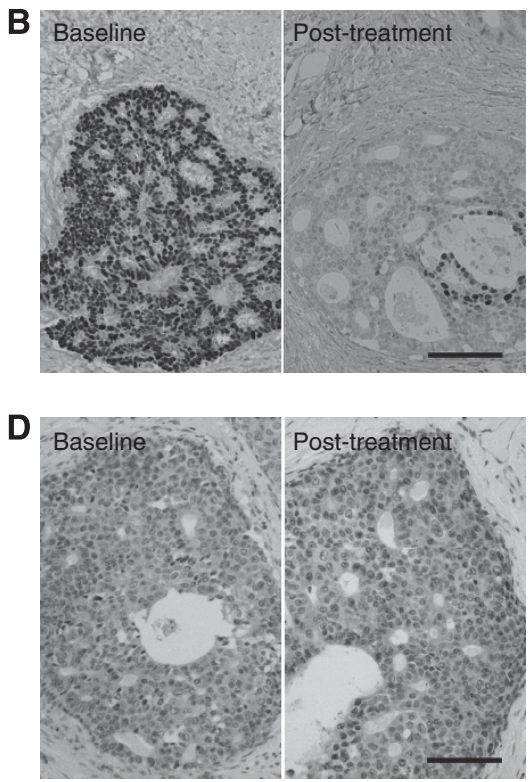

$\mathbf{F}$

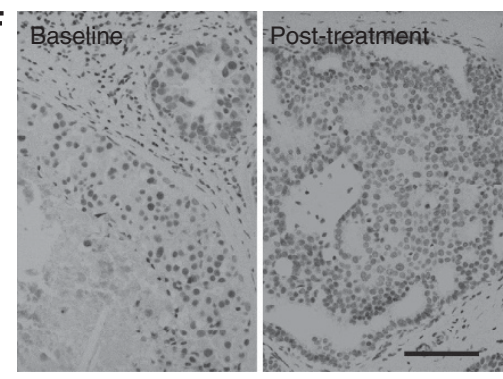

G

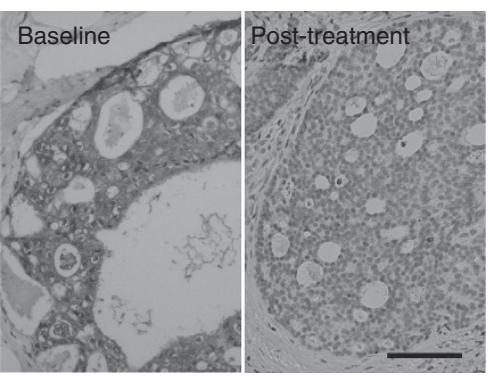

Figure 5. Immunohistochemistry for ER (A), PR (B), Ki-67 (C), aromatase (D), RBAP46 (E), C-MYB (F) and survivin (G) in DCIS at baseline (left panel) and corresponding post-treatment tissues (right panel). Left panels of (A) and (B), and right panels of (A) and (B) were of the same area, respectively. Bar $=100 \mu \mathrm{m}$, respectively.

aromatase activity in breast carcinoma tissues and the response to treatment with aromatase inhibitors (Bezwoda et al, 1987; Miller and O'Neill, 1987), and aromatase immunoreactivity reflected its enzymatic activity in human cancer cell lines (Suzuki et al, 1994). Ki-67 was reported as one of the biomarkers associated with subsequent breast carcinoma events after initial DCIS diagnosis (Kerlikowske et al, 2010), and Dowsett et al (2007) demonstrated that higher Ki-67 expression after endocrine therapy was significantly associated with poor recurrence-free survival in invasive breast carcinoma patients who received presurgical endocrine therapy for 2 weeks, whereas higher Ki-67 expression at baseline was not. Therefore, proliferation of ER-positive/ aromatase-positive DCIS may depend more on oestrogen actions compared with that of ER-positive/aromatase-negative cases. Further examinations are required to determine whether aromatase status at baseline may be a useful indicator of a response to aromatase inhibitor treatment or not in postmenopausal ER-positive DCIS patients.
US is known as a useful modality for preoperative evaluation of not only invasive breast carcinoma but also DCIS (Hayashi et al, 2012). In our study, the greatest dimension of DCIS evaluated by US was decreased after letrozole treatment, and it was significant (0.81-fold and $P=0.043$ ) in the longer treatment group (14-27 weeks) but not $(0.95$-fold and $P=0.85)$ in the shorter group (3-14 weeks). Most neoadjuvant studies with aromatase inhibitors in invasive breast carcinoma have treated patients for 3-4 months, and by this time many patients' tumours have responded sufficiently to downstage the surgical procedure required to excise the cancer (Dixon et al, 2007). In addition, Krainick-Strobel et al (2008) reported that a higher clinical response rate was observed after 8 months of neoadjuvant letrozole therapy compared with treatment for 4 months, and Takei et al (2011) suggested that the optimal duration of neoadjuvant aromatase inhibitor therapy was at least 4 months to obtain significant clinical response. Our present results were consistent with these data, and DCIS patients may also need $>4$ months of 


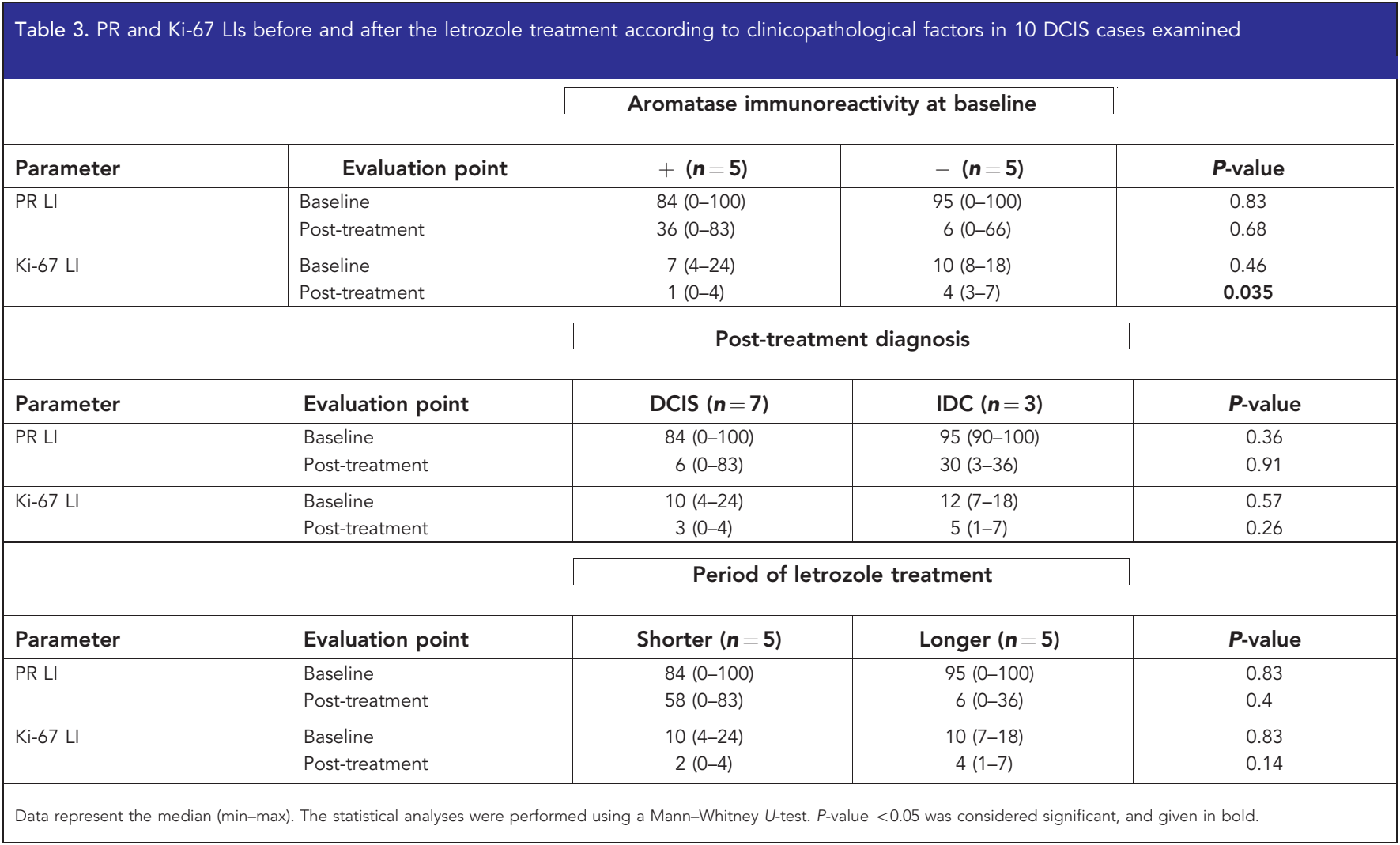

aromatase inhibitor treatment to obtain its clinical response. Our MR imaging examination frequently revealed high signal intensity within DCIS lesions after letrozole treatment. Strong high signal intensity on fat-suppressed T2-weighted imaging is rare in DCIS (Goto et al, 2012), and it may reflect oedematous stroma (Yuen et al, 2007) and/or accumulation of foam cells in DCIS tissues by letrozole treatment.

In our study, reduction of the tumour size in DCIS patients who received letrozole treatment seems much slower compared with the rapid decrement of intratumoral oestrogen levels, oestrogeninduced gene expressions and $\mathrm{Ki}-67 \mathrm{LI}$ in the DCIS tissues. Previous studies have demonstrated that apoptotic markers such as cleaved caspase 3 (Chen et al, 2009) and terminal deoxynucleotidyl transferase-mediated dUTP nick-end labelling deoxynucleotide labelling (Bundred et al, 2010), were not significantly changed regardless of aromatase inhibitor treatment in DCIS tissues. Therefore, apoptotic effects of aromatase inhibitors may not be evident in DCIS, which may be partly due to the delayed clinical responses to letrozole in DCIS patients in this study. It awaits further examinations in larger number of patients and longer duration of aromatase inhibitor treatment to clarify clinical significance of aromatase inhibitor in DCIS patients.

In summary, intratumoral concentrations of estrogens were significantly lower in DCIS tissues treated with letrozole compared with those without the therapy, and a great majority of oestrogeninduced genes showed lower expression levels in DCIS tissues after the therapy. Letrozole treatment significantly decreased Ki-67 LI and PR LI in DCIS tissues compared with the baseline, and the tumour size evaluated by US was significantly decreased in the longer duration group in this study (14-27 weeks). These results suggest that estrogens are mainly produced by aromatase in DCIS tissues, and aromatase inhibitors may potently inhibit the oestrogen-mediated growth and/or progression in postmenopausal ER-positive DCIS cases through rapid deprivation of intratumoral estrogens.

\section{ACKNOWLEDGEMENTS}

We appreciate the skillful technical assistance of Mr Katsuhiko Ono (Department of Anatomic Pathology, Tohoku University Graduate School of Medicine). This work was partly supported by Grant-in-Aid for Scientific Research (22590305) from the Japanese Ministry of Education, Culture, Sports, Science and Technology, and by an educational grant from Novartis Pharma (Tokyo, Japan).

\section{CONFLICT OF INTEREST}

The authors declare no conflict of interest.

\section{REFERENCES}

Bardou VJ, Arpino G, Elledge RM, Osborne CK, Clark GM (2003) Progesterone receptor status significantly improves outcome prediction over oestrogen receptor status alone for adjuvant endocrine therapy in two large breast cancer databases. J Clin Oncol 21: 1973-1979.

Barnes N, Haywood P, Flint P, Knox WF, Bundred NJ (2006) Survivin expression in in situ and invasive breast cancer relates to COX-2 expression and DCIS recurrence. Br J Cancer 94: 253-258.

Bezwoda WR, Mansoor N, Dansey R (1987) Correlation of breast tumour aromatase activity and response to aromatase inhibition with aminoglutethimide. Oncology 44: 345-349.

Bundred NJ, Cramer A, Morris J, Renshaw L, Cheung KL, Flint P, Johnson R, Young O, Landberg G, Grassby S, Turner L, Baildam A, Barr L, Dixon JM (2010) Cyclooxygenase-2 inhibition does not improve the reduction in ductal carcinoma in situ proliferation with aromatase inhibitor therapy: results of the ERISAC randomized placebo-controlled trial. Clin Cancer Res 16: 1605-1612.

Burstein HJ, Polyak K, Wong JS, Lester SC, Kaelin CM (2004) Ductal carcinoma in situ of the breast. N Engl J Med 350: 1430-1441. 
Chen YY, DeVries S, Anderson J, Lessing J, Swain R, Chin K, Shim V, Esserman LJ, Waldman FM, Hwang ES (2009) Pathologic and biologic response to preoperative endocrine therapy in patients with ER-positive ductal carcinoma in situ. BMC Cancer 9: 285.

Creighton CJ, Cordero KE, Larios JM, Miller RS, Johnson MD, Chinnaiyan AM, Lippman ME, Rae JM (2006) Genes regulated by oestrogen in breast tumour cells in vitro are similarly regulated in vivo in tumor xenografts and human breast tumors. Genome Biol 7: R28.

van Diest PJ, van der Wall E, Baak JP (2004) Prognostic value of proliferation in invasive breast cancer: a review. J Clin Pathol 57: 675-681.

Dixon JM, Faratian D, White S, Renshaw L, Murray J, Young O, Macaskill EJ, Williams L, Thomas J, Evans DB (2007) DCIS and aromatase inhibitors. J Steroid Biochem Mol Biol 106: 173-179.

Dowsett M, Ebbs SR, Dixon JM, Skene A, Griffith C, Boeddinghaus I, Salter J, Detre S, Hills M, Ashley S, Francis S, Walsh G, Smith IE (2005) Biomarker changes during neoadjuvant anastrozole, tamoxifen, or the combination: influence of hormonal status and HER-2 in breast cancer-a study from the IMPACT trialists. J Clin Oncol 23: 2477-2492.

Dowsett M, Smith IE, Ebbs SR, Dixon JM, Skene A, A'Hern R, Salter J, Detre S, Hills M, Walsh G. IMPACT Trialists Group (2007) Prognostic value of Ki67 expression after short-term presurgical endocrine therapy for primary breast cancer. J Natl Cancer Inst 99: 167-170.

Ebata A, Suzuki T, Takagi K, Miki Y, Onodera Y, Nakamura Y, Fujishima F, Ishida K, Watanabe M, Tamaki K, Ishida T, Ohuchi N, Sasano H (2012) Oestrogen-induced genes in ductal carcinoma in situ: their comparison with invasive ductal carcinoma. Endocr Relat Cancer 19: 485-496.

Erbas B, Provenzano E, Armes J, Gertig D (2006) The natural history of ductal carcinoma in situ of the breast: a review. Breast Cancer Res Treat 97(2): $135-144$.

Frasor J, Danes JM, Komm B, Chang KC, Lyttle CR, Katzenellenbogen BS (2003) Profiling of estrogen up- and down-regulated gene expression in human breast cancer cells: insights into gene networks and pathways underlying estrogenic control of proliferation and cell phenotype. Endocrinology 144: 4562-4574.

Geisler J, Detre S, Berntsen H, Ottestad L, Lindtjørn B, Dowsett M, Einstein Lønning P (2001) Influence of neoadjuvant anastrozole (Arimidex) on intratumoral estrogen levels and proliferation markers in patients with locally advanced breast cancer. Clin Cancer Res 7: 1230-1236.

Geisler J, Helle H, Ekse D, Duong NK, Evans DB, Nordbø Y, Aas T, Lønning PE (2008) Letrozole is superior to anastrozole in suppressing breast cancer tissue and plasma estrogen levels. Clin Cancer Res 14: 6330-6335.

Goto M, Yuen S, Akazawa K, Nishida K, Konishi E, Kajihara M, Shinkura N, Yamada K (2012) The role of breast MR imaging in pre-operative determination of invasive disease for ductal carcinoma in situ diagnosed by needle biopsy. Eur Radiol 22: 1255-1264.

Hayashi N, Tsunoda H, Abe E, Kikuchi M, Enokido K, Tsugawa K, Suzuki K, Nakamura S (2012) Ultrasonography- and/or mammography-guided breast conserving surgery for ductal carcinoma in situ of the breast: experience with 87 lesions. Breast Cancer 19: 131-137.

Kerlikowske K, Molinaro AM, Gauthier ML, Berman HK, Waldman F, Bennington J, Sanchez H, Jimenez C, Stewart K, Chew K, Ljung BM, Tlsty TD (2010) Biomarker expression and risk of subsequent tumors after initial ductal carcinoma in situ diagnosis. J Natl Cancer Inst 102: 627-637.

Krainick-Strobel UE, Lichtenegger W, Wallwiener D, Tulusan AH, Jänicke F, Bastert G, Kiesel L, Wackwitz B, Paepke S (2008) Neoadjuvant letrozole in postmenopausal estrogen and/or progesterone receptor positive breast cancer: a phase IIb/III trial to investigate optimal duration of preoperative endocrine therapy. BMC Cancer 8: 62.

Mehta RJ, Jain RK, Leung S, Choo J, Nielsen T, Huntsman D, Nakshatri H, Badve S (2011) FOXA1 is an independent prognostic marker for ER-positive breast cancer. Breast Cancer Res Treat 131: 881-890.

Miller WR, O'Neill J (1987) The importance of local synthesis of estrogen within the breast. Steroids 50: 537-548.
Robanus-Maandag EC, Bosch CA, Kristel PM, Hart AA, Faneyte IF, Nederlof PM, Peterse JL, van de Vijver MJ (2003) Association of C-MYC amplification with progression from the in situ to the invasive stage in C-MYC-amplified breast carcinomas. J Pathol 201: 75-82.

Shibuya R, Suzuki T, Miki Y, Yoshida K, Moriya T, Ono K, Akahira J, Ishida T, Hirakawa H, Evans DB, Sasano H (2008) Intratumoral concentration of sex steroids and expression of sex steroid-producing enzymes in ductal carcinoma in situ of human breast. Endocr Relat Cancer 15: 113-124.

Steeg PS, Zhou Q (1998) Cyclins and breast cancer. Breast Cancer Res Treat 52: $17-28$.

Suzuki S, Takagi K, Miki Y, Onodera Y, Akahira J, Ebata A, Ishida T, Watanabe M, Sasano H, Suzuki T (2012) Nucleobindin 2 in human breast carcinoma as a potent prognostic factor. Cancer Sci 103: 136-143.

Suzuki T, Inoue A, Miki Y, Moriya T, Akahira J, Ishida T, Hirakawa H, Yamaguchi Y, Hayashi S, Sasano H (2007) Early growth responsive gene 3 in human breast carcinoma: a regulator of estrogen-meditated invasion and a potent prognostic factor. Endocr Relat Cancer 14: 279-292.

Suzuki T, Miki Y, Nakamura Y, Moriya T, Ito K, Ohuchi N, Sasano H (2005) Sex steroid-producing enzymes in human breast cancer. Endocr Relat Cancer 12: 701-720.

Suzuki T, Sasano H, Sasaki H, Fukaya T, Nagura H (1994) Quantitation of P450 aromatase immunoreactivity in human ovary during the menstrual cycle: relationship between the enzyme activity and immunointensity. J Histochem Cytochem 42: 1565-1573.

Takagi K, Miki Y, Nagasaki S, Hirakawa H, Onodera Y, Akahira J, Ishida T, Watanabe M, Kimijima I, Hayashi S, Sasano H, Suzuki T (2010) Increased intratumoral androgens in human breast carcinoma following aromatase inhibitor exemestane treatment. Endocr Relat Cancer 17: 415-430.

Takagi K, Miki Y, Shibahara Y, Nakamura Y, Ebata A, Watanabe M, Ishida T, Sasano H, Suzuki T (2013b) BUB1 immunolocalization in breast carcinoma: its nuclear localization as a potent prognostic factor of the patients. Horm Cancer 4: 92-102.

Takagi K, Moriya T, Kurosumi M, Oka K, Miki Y, Ebata A, Toshima T, Tsunekawa S, Takei H, Hirakawa H, Ishida T, Hayashi S, Kurebayashi J, Sasano H, Suzuki T (2013a) Intratumoral estrogen concentration and expression of estrogen-induced genes in male breast carcinoma: comparison with female breast carcinoma. Horm Cancer 4: 1-11.

Takei H, Kurosumi M, Yoshida T, Hayashi Y, Higuchi T, Uchida S, Ninomiya J, Oba H, Inoue K, Nagai S, Tabei T (2011) Neoadjuvant endocrine therapy of breast cancer: which patients would benefit and what are the advantages? Breast Cancer 18: 85-91.

Wiechmann L, Kuerer HM (2008) The molecular journey from ductal carcinoma in situ to invasive breast cancer. Cancer 112: 2130-2142.

Wolff AC, Hammond ME, Schwartz JN, Hagerty KL, Allred DC, Cote RJ, Dowsett M, Fitzgibbons PL, Hanna WM, Langer A, McShane LM, Paik S, Pegram MD, Perez EA, Press MF, Rhodes A, Sturgeon C, Taube SE, Tubbs R, Vance GH, van de Vijver M, Wheeler TM, Hayes DF. American Society of Clinical Oncology; College of American Pathologists (2007) American Society of Clinical Oncology/College of American Pathologists guideline recommendations for human epidermal growth factor receptor 2 testing in breast cancer. J Clin Oncol 25: 118-145.

Yuen S, Uematsu T, Kasami M, Tanaka K, Kimura K, Sanuki J, Uchida Y, Furukawa $H$ (2007) Breast carcinomas with strong high-signal intensity on T2-weighted MR images: pathological characteristics and differential diagnosis. J Magn Reson Imaging 25: 502-510.

This work is published under the standard license to publish agreement. After 12 months the work will become freely available and the license terms will switch to a Creative Commons AttributionNonCommercial-Share Alike 3.0 Unported License.

Supplementary Information accompanies this paper on British Journal of Cancer website (http://www.nature.com/bjc) 